\title{
Metal-Organic Frameworks: A promising material for enhancing the photovoltaic performance of Dye-Sensitized Solar Cells
}

\author{
${ }^{1}$ Sandeep Kumar Sharma \\ ${ }^{1}$ Research Scholar, DeshBhagat University, MandiGobindgarh, Punjab-India \\ E-mail: sandeep.puchd07@gmail.com
}

\begin{abstract}
The introgression of metal-organic frameworks (MOFs) in dye-sensitized solar cells has received greater attention over the past decade. Many efforts have been made to improve the performance of these cells by optimizing itsdifferent components viz.photosensitizer, photoanode, and counter electrode. This article provides the recent advances in each of these major directions achieved through MOF and its hybrid components in dye-sensetized solar cells.
\end{abstract}

Keywords:DSSCs, MOFs andphotoanode

\section{Introduction:}

Dye-Sensitized Solar Cells (DSSCs) have attracted researcher's interest in commercializing a convenient renewable energy source which is environmentally friendly and cost-effective. The successful exploitation of solar energy for its efficient conversion into electrical energy follows the landmark development of Dye-Sensitized Solar Cells (DSSCs) by Brian O’ Regan and Michael Gratzel[1]. Significant performance of these DSSCs under indoor light conditions,environment friendliness, easy fabrication and cost-effectiveness has encouraged researchers to explore their possibility in commercializing this as an efficient energy source. Many materials have been tested to improve the overall device performance leading to efficiencies over 14 per cent. Regular studies are being undertaken to improve the overall performance of these photovoltaic cells. Metal-Organic Frameworks (MOFs) are one such new class of materials which because of their attractive properties viz. high porosity, flexible fabrication, high thermal and chemical stability and good light-harvesting properties offer its utilization as different components of DSSCs. Further, the doping and hybridization of conventional MOFs with various conducting materials like carbon nanotubes and graphene, etc have added to their potential in successful exploitation of them in generating the next generation improved photovoltaic cells with enhanced efficiency and cost-effectiveness.

MOFs are the metal cations or clusters which are linked with organic ligands through strong coordinate bonds [2]. They were first time referred as open microporous crystalline framework obtained 
between Cu-metal and 4, 4-bipyradine ligand via hydrothermal synthesis [3].These MOFs furnish many attractive properties viz., high thermal and chemical stability, ability of changing chemical properties and high porosity etc. [4].Moreover, their interesting optical properties, flexible fabrication and highly porous nature have added to their use as potential component in the development of next generation DSSCs [5-8]. Many applications of MOFs showing enhanced photovoltaic performance in various DSSCs have been recorded by different workers [4-5, 9-11]. However, most of the conventional MOFs suffer from a limitation that they exhibit insulating character which is attributed to their open porous structure that hinders electron free flow and hence disable charge retention.Thus their exploitation in photovoltaics needs the successful incorporation of conductivity in them [12]. In view of it, MOFs are doped and hybridized with various conducting materials [8, 13-18]. Moreover, Graphene based MOFs are one such potential hybrid which offers its advantage over the traditional MOFs and are continuously attracting the researcher interest [19, 20]This article selectively focuses on the developments and advancements made in the application of MOFs and MOF-composites as counter electrode, photoanode, electrolyte and photosensitizer component in the fabrication of next generation DSSCs.

\section{MOFs in DSSCs: literature review}

The application of MOFs in photovoltaics has been reported to offer many important advantages, like the use of MOFs as photoanode and sensitizer can effectively improve the open circuit potential and thereby resulting in enhanced cell efficiency. The landmark initial work exploiting the use of MOFs as photoanode in improving the performance of photovoltaics involved a study that developed a zeolite imidazolate framework, ZIF-8 (a well-known subfamily of MOFs), generated through the supramolecular self-assembly of $\mathrm{Zn}^{2+}$ and 2-methyl-imidazole and its coating on a $\mathrm{TiO}_{2}$ electrode and later its fabrication in DSSC, proved promising in enhancing its open-circuit voltage, $V_{o c}$ ranges from 756-796 V at different growth times compared to Platinum (P25) electrode where $V_{o c}$ was $741 \mathrm{~V}$ [5]. However, this work suffered from the limitation that the short-circuit photocurrent $\left(J_{s c}\right)$ and the power conversion efficiency values decreased with the increasing ZIF-8 growth time. The advancement in this aspect was made wherein an effective strategy that modify this MOF component involved the posttreatment approach with optimization of ZIF-8 by surface treatment followed by its treatment with $\mathrm{TiO}_{2}$ photoanode and this strategy improved the overall performance of the cell and overcome the problem of severely decreased short-circuit photocurrent as in the earlier report [21]. This study optimized the best conditions for the photovoltaic properties which can be achieved when the growth time of ZIF-8 was 7 minutes and the $\mathrm{TiO}_{2}$ photoanode was post-treated for 2 hours. The decrease in $J_{s c}$ was avoided in this 
post-treatment approach because of the tightly absorbed dyes on $\mathrm{TiO}_{2}$ photoanode. Copper $(\mathrm{Cu})$-based MOFs also came up as the potential precursor in the improvement of cell performance especially as a sensitizer component. A typical study demonstrated that a $\mathrm{TiO}_{2}$-based solar cell which when fabricated using a iodine doped Cu-MOF (Copper (II) benzene 1,3,5-tricarboxylate) improved the cell performance $\left(J_{s c}=1.25 \mathrm{~mA} \mathrm{~cm}{ }^{-2} \&\right.$ efficiency $\left.=0.26 \%\right)$ under the illumination of 1 sun radiation, thereby suggesting the successful exploitation of iodine doped Cu-MOFs as a light absorbing material [7]. The replacement of $\mathrm{Cu}-\mathrm{MOFs}$ with Ruthenium $(\mathrm{Ru})$-MOFs further improved the device performance. In a study, a thin layer of iodine doped $\mathrm{Ru}$ based MOFs were synthesized using a layer-by-layer technique and a $\mathrm{TiO}_{2}$ mesoporous film was sensitized over this iodine doped Ru-MOF in a Gratzel type liquid junction solar cell which demonstrated the enhanced efficiency of 1.22 per cent and $J_{s c}$ of $2.51 .25 \mathrm{~mA} \mathrm{~cm}^{-2}$ [22].

Later in the same year, the introduction of carbon nano-tubes into a $\mathrm{TiO}_{2}$ nanoparticle film and its sensitization with Cu-based MOF has further added to the arena of advancements in the development of the solar cell with accelerated pholovoltaic performance [23].In their study, they made a composite of $\mathrm{TiO}_{2}$ nanoparticle and multi-walled carbon nanotubes (MWCNTs) which were sensitized with Cu-based MOFs using a layer-by-layer deposition technique and this cell assembly witnessed an enhanced photovoltaic performance (60\% increased PCE). This increase in cell performance was attributed to the accelerated electron transfer rate that resulted due to the addition of MWCNTs which improved the interfacial charge transfer resistance. Furthermore, the generation of $\mathrm{TiO}_{2}$ from $\mathrm{MOFs}$ and its utilization in photovotaics improved the efficiency. In a study made by Chi et al [24], the shape and morphologycontrolled MIL-125 (Ti) (Materials Institute Lavoisier), a subclass of MOFs was produced using poly(ethylene glycol)diglycidyl ether (PEGDGE) and this was further calcinated to generate a mesoporous hierarchical $\mathrm{TiO}_{2}$ deposited onto a nanocrystalline (nc) $\mathrm{TiO}_{2}$ layer of a DSSC. This DSSC fabricated with hier- $\mathrm{TiO}_{2} / \mathrm{nc}-\mathrm{TiO}_{2}$ bilayer photoanode and a quasi-solid-state polymer electrolyte exhibited as high as 7.0 per cent efficiency compared to the nc- $\mathrm{TiO}_{2}$ monolayer photoanode based DSSC where the respective value was only 4.6 per cent. Similarly, the efficiency of solid stated DSSCs reached as high as 8.0 per cent. This improvement in light harvesting efficiency of cell was attributed to their dual functional properties viz. ability to reflect light back into the dye as well as their high surface area that ensured high dye loading.In a similar study, MIL-125 (Ti), was utilized to generate a porous anatase $\mathrm{TiO}_{2}$ and its fabrication in DSSC as a photoanodic component exhibited an higher efficiency of 7.20 per cent than that of P25, which was ascribed to the enhanced dye absorption, faster electron transport and better charge collection efficiency [10]. 
Extending to the utilization of carbon nanotubes as done in previous work made by Lee et al[23], Cui et al [25] utilized cobalt disulfide $\left(\mathrm{CoS}_{2}\right)$ embedded carbon nanotubes sulfurized with ZIF-67 MOF (Zeoliticimidazolate framework 67, $\mathrm{Co}(\mathrm{min})_{2}, \mathrm{~min}=2$-methylimidolate) as counter electrode (CE) in a DSSC thatachieved significantly better efficiency. Here, the sulfurization time of $\mathrm{CoS}_{2}$ was optimized to maximize the efficiency of DSSC and it was found that the DSSC based on $\mathrm{CoS}_{2}$ embedded carbon nanotubes which was sulfurized for 4 hrs exhibited the highest PCE of 8.2 per cent compared to other $\mathrm{CoS}_{2} \mathrm{CEs}$ and Pt-based DSSC, where it was 7.88 per cent.This significant improvement was because of the synergic effect of inner $\mathrm{CoS}_{2}$ nano particles and an amorphous carbon matrix which resulted in high catalytic activity, good electrical conductivity with excellent durability. In the later year, Spoerkeet al [8] made an attempt to define the role of incorporated MOFs and its potential effect of residual MOF precursor in the improvement of a DSSC. They reported that the dispersed nanocrystals of a pillared porphyrin framework, PPF-4 (one of the series of MOFs) when assembled and characterized as a sensitizer in a $\mathrm{TiO}_{2}$ fabricated DSSC resulted in significant reduction in interfacial charge transfer resistance, contributing to the overall improved device performance.

Polyoxometalates (POMs) are another class of MOFs that have been used in DSCs being good electron acceptors and offers improvement in the charge separation within a device. In a recent study made by Zhang et al [26], a novel Fe-based polyoxometalates@ metal organic framework (POM@MOF (Fe)) combined with $\mathrm{ZnO}$ was synthesized, assembled and characterized as photoanode and the results showed that the photovoltaic efficiency of this composite based solar cell increased by 28 per cent compared to the bare $\mathrm{ZnO}$, suggesting that this Fe-based polyoxometalates modified $\mathrm{ZnO}$ could be a promising photoanode candidate in solar cells. The enhanced efficiency of this composite was ascribed to the good light absoption in visible range and better electron-hole separation properties of this Febased polyoxometalate MOF.

Another research area in the development of improved photovoltaic cells exploited the potential utilization of Surface-anchored metal-organic frameworks (SURMOFs) that showed controlled growth orientation, regular monolithic porous crystalline frameworks. However, the use of these SURMOFs for solar energy conversion in particular, DSSC, is challenging dueto the poor solar capture ability and photo-induced charge carrier mobility. An attempt had been made in this aspect by Ahmad et al [18], where triplet-triplet annihilation up-conversion (TTA-UC) technique was utilized to fabricate a prototype Zn-perylene SURMOF material which was integrated with metal-free Bodipy sensitizer and glassy PMMA [poly(methyl methacrylate)] polymer and the results revealed thatthis $\mathrm{TiO}_{2}-\mathrm{Zn}$ - 
peryleneSURMOF-Bodipy/PMMA, when used as a photoanode exhibited the best performances among the devices using $\mathrm{TiO}_{2}$-Zn-perylene SURMOF, $\mathrm{TiO}_{2}$-Bodipy/PMMA, and $\mathrm{TiO}_{2}$ as photoanode. It has beendemonstrated that the photocurrent can considerably be enhanced via TTA-UC due the ZnperyleneSURMOF-Bodipy/PMMA materials-based devices. Although the device performance was not very impressive, in this work, but it demonstrated thestrategy with the upconverting SURMOF-based system for solar energy conversion. Another new class of $\mathrm{TiO}_{2}$ aerogel-MOF ([Zn(N-(4-pyridylmethyl)L-valine $\mathrm{HCl})(\mathrm{Cl})]\left(\mathrm{H}_{2} \mathrm{O}\right)_{2}$ )nanocomposite was synthesizedby sol-gel method and was successfully fabricated as a photoanode component ofa quasi-solid DSSCs which subsequently achieved as high as 2.34 per cent power conversionefficiency andshort circuit current density of $6.22 \mathrm{mAcm}^{-2}$. This improved cell performance was attributed to the high surface area of thenanocomposite material that enhanced the amount of dye adsorptionas well as the short-circuit current density [27].

Besides many metal ions being used in the doping and hybridization of MOFs, graphene also could be used to enhance the efficiency of MOFs for its utilization in energy conversion especially in DSSCs. Owing to the fascinating properties of graphene, such as huge mechanical strength, high thermal conductivity, superior transparency, large specific surface area and exceptional charge transport properties[28, 29], it offersthe potential of its utilization to generate the MOF hybrids that could be used for energy conversion. Many MOF/graphene composites were found promising in improving the overall performance of these solar cells. The application of a graphene-MOF composite as a potential photosensitizer material in DSSC and its photoanodic role was investigated by generating an electrochemical assembly of Europium $(\mathrm{Eu})$ and benzene 1,3,5-tricarboxylate with graphene (EuMOF/graphene composite) on a $\mathrm{TiO}_{2} / \mathrm{FTO}$ substrate and this hybrid MOF when substituted as photoanode improved the basic performance of the cell (PCE $=2.3 \%$ ) over the traditional metal-based (Cu-MOF) and carbon nanotube based DSSC [19].This proposed formation of graphene-MOF thin films by electrolyticdeposition resulted in a novel homogenous surface with low inter-facial resistance that was responsible for the overall improvement of the cell.In continuation to this study, another attempt utilizing gaphene-MOFs had been made by Sharma et al [20], where, the hybridization of benzoic acid functionalized graphene (BFG) with Eu-BTB MOF (BTB = 1,3,5-tris(4-carboxyphenyl) benzene) demonstrated the successful characterization and application of this composite as photoanodic component of a DSSC which achieved as high as 4.5 per cent efficiency compared to 2.35 per cent obtained from the reference graphene only cell.This better performance of Eu-BTB/BFG electrode was ascribed to the combination of enhanced photoactive characteristics of the composite (from Eu-BTB) along with thedesirable electronic conductivity (from graphene). Also, the porous structure of the Eu- 
BTB minimized the charge recombination losses and hence provided anefficient charge transfer path whichresultedin anincreased short circuit current density from Eu-BTB/BFG photoanode in comparison to the graphene-onlyphotoanode.

Besides their application as photoanode and sensetizer, MOFs have been considered useful in the fabrication of counter electrodes as their larger surface area and better roughness than the conventional Pt counter electrodes allow the development of ecofriendly panels. Keeping in view of the above stated fact of MOFs, a study has been made by Hsu et.al. [11], where they synthesized CoS nanoparticlesthrough the CTAB-(cetyltrimethylammonium bromide) assisted preparation of a metalorganic framework, ZIF-67, which was subsequentlyoxidized and sulfurized to CoS and these generated CoS nanoparticles were thencoated onto FTO substrates and fabricated as counter electrodefor DSSCs. This Pt-free counter electrode comprising of MOF (ZIF-67) derived Cobalt sulfide (CoS) nanoparticle evidenced an improved efficiency of 8.1 per cent which was comparable to that of Pt-based DSSC, thereby indicating its potential in substituting the Pt for generating economic next generation photovoltaic cells. The improved performance of this Pt-free DSSC was attributed to the MOF derived CoS nanoparticle, whose nanosize enhanced interaction withdye molecules and increased roughness factor that further increased the $V_{O C}$ value and thereby the fill factor value. In another study made by Xieet al [17], the bimetallic MOF (Co/Ni) was used to generate a Co/Ni alloy@carbon nanotubes embedded carbon nanocages (Co/Ni@CNTs-C), which was characterized as counter electrode component in a commercial DSSC and exhibited a remarkable PCE (9.04\%) compared to the traditional Pt counter electrode based cell (7.88\%).Further, this CoNi@CNTs-C-200 CE also exhibited much better electrochemical stability than conventional Pt CE during long-term electrochemical cycling, which formed a strong base for the practical application in high-performance DSSCs.Thus, these MOF derivedCoNi@CNTs-Coffersits great potential as a successful low-cost, stable and efficient CE material for next-generation DSSCs. In a similar study, a bimetallic Co-Ni MOF was calinated to generate a novel Ni-Co alloy@carbon microspheres which had shown excellent electrocatalytic activity and conductivity when fabricated as a counter electrode component of a DSSC and a high PCE of 9.3 per cent. ${ }^{30}$ Anotherpromising material to replace the expensive Ptin DSSCs, especially for the indoor application is thezinc selenide (ZnSe). A study demonstrated the successful synthesis of ZIF-7 derived zinc selenide (ZnSe) nanocompositewhich was hybridized with a N-doped carbon (NC) to develop a ZIF-ZnSe-NC composite and this when used as counter electrode improved the photovoltaic performance of the cell (PCE $=8.69 \%)$ [33]. This study thus offered a new strategy for the applicationof ZIFs as precursors in the field of photovoltaic devices. Further, Xuet al [32] utilized ZIF as counter 
electrode component to fabricate quantum dot sensitized solar cells. Here, a ZIF-67-derived thin filmwas coated on the conducting glass followed by its calcination through a series of carbonization and sulfuration treatments and this resultant MOF composite when served as a counter electrode achieved as high as 3.77 per cent light-to-electric conversion efficiency.Another work providing a new strategy to design promising PtfreeCE materials with both low cost and high efficiency involved thedevelopment of a new generation DSSC which was fabricated with a counter electrode based on nitrogen doped carbon (CN) MOF i.e. cobalt oxide $\left(\mathrm{Co}_{3} \mathrm{O}_{4}\right)$, tungsten carbide (WC) and reduced graphene oxide (rGO)nanocomposite material $\left(\mathrm{Co}_{3} \mathrm{O}_{4}-\mathrm{WC}-\mathrm{CN} / \mathrm{rGO}\right)$, which achieved as high as 7.38 per cent PCE offering its superiority over the traditional Pt based cell (PCE =6.85\%). This enhanced efficiency was ascribed to the synergisticeffect of $\mathrm{Co}_{3} \mathrm{O}_{4}$ and WC nanoparticles and high electrical conductivity of rGO [33].

Most of the developments were made in respect of utilization of MOFs as photoanode, sensetizer and counter electrode components of the DSSCs attributing to their efficient light harvesting properties. However, some studies also pertain to the utilization of MOFs as electrolyte component offering improved photovoltaic performance of solar cells. Like in a work, where a polymer composite containing a Mg-based MOF when served as an electrolyte for quasi-solid DSSC resulted in remarkable solar energy conversion efficiency (4.8\%) [34].However, their developments as an electrolyte need to be explored.

3. Conclusion:The tunable properties of MOFs viz. efficient light harvesting, flexible fabrication and highly porous nature can be successfully incorporated for the improvement of overall photovoltaic performance of DSSCs.However, the poor electrical conductivity of MOFs(insulators or weak semiconductors) and their large particle size limits their application in photovotaics.Advancements have been madein development of modified MOFs or MOF composites generatedthrough hybridization and doping with many metals, carbon nanotubes and graphene, which offered their successful utilization as different components of DSSCs with improved overall cell performance. Keeping in view the desired properties of MOFs that impact device performance,future studiesare to be established for its robust structure-property relationships, design and synthesis of MOF-based DSSCs that will converge to desired commercialization goals.

4. Conflict of interest: There is no conflict of interest to declare.

5. References: 
[1] B. O’Regan and M. Gratzel,“A low-cost, high-efficiency solar cell based on dye-sensitized colloidal $\mathrm{TiO}_{2}$ films,”Nature.,vol. 353,(1991), pp.737-740.

[2] H. C.Zhou, J. R. Longand O. M. Yaghi, "Introduction to metal-organic frameworks,” Chem. Rev.,vol. 112, (2012), pp. 673-674.

[3] O. M.Yaghi and H. Li, "Hydrothermal synthesis of a metal-organic framework containing larger rectangular channels,” J. Am. Chem. Soc.,vol. 117(1995), pp.10401-10402

[4] I. R. Perera, C. V.Hettiarachchiand R. J. K. U. Ranatunga, "Metal-Organic Frameworks in DyeSensitized Solar Cells,"Edited H. Tyagi et al. in Advances in Solar Energy Research, Energy, Environment, and Sustainability, Springer Nature Singapore Pte Ltd., (2019)p.175.

[5] Y. Li, A. Pang, C. Wang and M. Wei, “ Metal-organic frameworks: promising materials for improving the open circuit voltage of dye-sensitized solar cells,”J. Mater. Chem., vol. 21, (2011), pp.17259-17264

[6] C. Y. Lee, O. K. Farha, B. J. Hong, A. A. Sarjeant, S. T. Nguyen and J. T. Hupp, “Lightharvesting metal-organic frameworks (MOFs): efficient strut-to-strut energy transfer in bodipy and porphyrin-based MOFs,”J. Am. Chem. Soc.vol. 133, (2011),pp. 15858-15861.

[7] D. Y. Lee, D. V. Shinde,S. J. Yoon,K. N Cho, W.Lee, N. K. Shrestha and S. H. Han, “Cu-based metal-organic frameworks for photovoltaic application,”J. Phys. Chem. C., vol. 118(2014a), pp.16328-16334.

[8] E. D. Spoerke, L. J. Small, M. E. Foster, J. Wheeler, A. M. Ullman, V. Stavila, M. Rodriguez and M. D. Allendorf, "MOF-sensitized solar cells enabled by a pillared porphyrin framework,"J. Phys. Chem. C., vol. 121, (2017), pp.4816-4824

[9] Y. Li, Z. Che, X. Sun, J. Dou and M. Wei, “Metal-organic framework derived hierarchical ZnO parallelepipeds as an efficient scattering layer in dye-sensitized solar cells,”Chem. Commun.,vol. 50, (2014), pp.9769-9772

[10] J. Dou, Y. Li, F. Xie, X. Ding and M.Wei, "Metal-organic framework derived hierarchical porous anatase $\mathrm{TiO}_{2}$ as a photoanode for dye-sensitized solar cell,”Cryst. Growth. Des., vol.16,(2016), pp. 121-125

[11] S. H. Hsu, C. T. Li, H. T. Chien, R. R. Salunkhe, N. Suzuki, Y. Yamauchi, K. C. Ho and K. C. W. Wu, "Platinum-free counter electrode comprised of metal-organic-framework (MOF)derived cobalt sulfide nanoparticles for efficient dye-sensitized solar cells (DSSCs),”Sci. Rep., vol. 4,(2014), p. 6983. 
[12] A. A. Talinet al., "Tunable electrical conductivity in metal-organic framework thin-film devices,”Science., vol. 343 (2014), pp. 66-69

[13] W. W. Zhan, Q. Kuang,J. Z. Zhou, X. J. Kong, Z. X. Xieand L. S. ZhengL, “Semiconductor@metal-organic framework core-shell heterostructures: a case of ZnO@ZIF-8 nanorods with selective photoelectrochemical response,”J. Am. Chem. Soc., vol.135, (2013), pp.1926-1933.

[14] T. Neumann et al., "Superexchange charge transport in loaded metal organic frameworks ACS,"Nano., vol. 10, (2016), pp. 7085-7093.

[15] X. Sun, Y. Li, J. Dou, D. Shen, M. Wei, "Metal-organic frameworks derived carbon as a high-efficiency counter electrode for dye-sensitized solar cells,”J. Power Sources,vol. 322, (2016), pp.93-98.

[16] R. Tang, Z. Xie, S. Zhou, Y. Zhang, Z. Yuan, L. Zhang and L. Yin, " $\mathrm{Cu}_{2} \mathrm{ZnSnS}_{4}$ nanoparticle sensitized metal-organic framework derived mesoporous $\mathrm{TiO}_{2}$ as photoanodes for high-performance dye-sensitized solar cells,” ACS Appl.Mater. Interface., vol.8, (2016), pp.22201-22212

[17] Z. Xie, X. Cui, W. Xu and Y. Wang, "Metal-Organic Framework Derived CoNi@CNTs Embedded Carbon Nanocages for Efficient Dye-Sensitized Solar Cells,”Electrochim. Acta., vol. 229, (2017), pp.361-370.

[18] S. Ahmad, J. Liu, W. Jiand L. Sun, "Metal-Organic Framework Thin Film-Based Dye Sensitized Solar Cells with Enhanced Photocurrent,” Materials., vol.11, (2018), p.1868.

[19] R. Kaur, K. H. Kim and A. Deep, “A convenient electrolytic assembly of graphene-MOF composite thin film and its photoanodic application,”Appl. Surf.Sci,.vol. 396, (2017), pp.13031304

[20] S. K. Sharma, H. Kaur, K. Kumar, M. Tyagiand A. Deep, “Synthesis and solar cell application of a composite of Eu-BTB MOF with functionalized graphene,”Mater. Res. Express., vol.5, (2018), p.125501.

[21] Y. Li, Z. Che, X. Sun, J. Douand M. Wei, "Metal-Organic Frameworks at Interfaces in Dye-Sensitized Solar Cells,”Chem. Sus. Chem., vol. 7, (2014), p.2469.

[22] D. Y. Lee, E. K. Kim, C. Y. Shin, D. V. Shinde, W. Lee, N. K. Shrestha, J. K. Leeand S. H. Han, "Layer-by-layer deposition and photovoltaic property of Ru-based metal-organic frameworks,"RSC. Adv., vol.4,(2014b), p.12037. 
[23] D. Y. Lee, C. Y. Shin, S. J. Yoon, H. Y. Lee, W. Lee, N. K. Shrestha, Lee J K and S. H. Han, "Enhanced photovoltaic performance of Cu-based metal-organic frameworks sensitized solar cell by addition of carbon nanotubes,”Sci. Rep,vol.4, (2014c), p.3930.

[24] W. S. Chi, D. K. Roh, C. S. Lee, J. H. Kim, “A shape-and morphology-controlled metal organic framework template for high-efficiency solid-state dye-sensitized solar cells,”J. Mater. Chem. A., vol.3, (2015), pp.1599-15608.

[25] X. Cui, Z. Xieand Y.Wang, "Novel $\mathrm{CoS}_{2}$ embedded carbon nanocages by direct sulfurizing metal-organic frameworks for dye-sensitized solar cells,”Nanoscale, vol.8, (2016), pp.11984-11992.

[26] W. Zhang, W. Li, X. He, L. Zhao,H. Chen, L. Zhang, P. Tian, Z. Xin, W. Fang and F. Zhang, "Dendritic Fe-based polyoxometalates @ metal-organic framework (MOFs) combined with ZnO as a novel photoanode in solar cells,” J. Mater. Sci. Mater.inElectronics., vol. 29, (2018), pp.1623-1629.

[27] S. Alwin, V. Ramasubbu and X. S. Shajan, “ $\mathrm{TiO}_{2}$ aerogel-metal organic framework nanocomposite: a new class of photoanode material for dye-sensitized solar cell applications,”Bull. Mater. Sci., vol.41, (2018), p.1827.

[28] K. S. Novoselov, A. K. Geim, S. V. Morozov, D. Jiang, Y. Zhang, S. V. Dubonos, I. V. Grigorievaand A. A. Firsov, "Electric field effect in atomically thin carbon films Science," vol. 306, (2004), pp. 666-669.

[29] M. J. Allen, V. C. Tungand R. B. Kaner, "Honeycomb carbon: a review of graphene,”Chem. Rev., vol.110, (2010), pp. 132-145.

[30] X. Jiang, H. Li, S. Li,S. Huang, C. Zhu andL. Hou,“Metal-organic framework-derived Ni-Co alloy@carbon microspheres as high-performance counter electrode catalysts for dyesensitized solar cells,”Chem. Eng. J., vol. 334, (2018), pp.8419-8431.

[31] S. L. Jian, Y J. Huang, M. Yeh and K. C. Ho,“A zeoliticimidazolate framework-derived ZnSe/ N-doped carbon cube hybrid electrocatalyst as the counter electrode for dye-sensitized solar cells,”J. Mater. Chem. A.,vol.6,(2018), pp. 5107-5118

[32] W. Xu,Y. Sun,B. Ding andJ. Zhang, "Zeolitic-imidazolate frameworks derived Pt-free counter electrodes for high-performance quantum dot-sensitized solar cells,"R. Soc. Open Sci., vol.5, (2018), p.180335. 
[33] L. Chen, W. Chen andE. Wang, "Graphene with cobalt oxide and tungsten carbide as a low-cost counter electrode catalyst applied in Pt-free dye-sensitized solar cells,”J. Power. Sources.,vol.380,(2018), pp.18-25.

[34] F. Bella,R. Bongiovanni,R S. Kumar,M. A. KulandainathanandA. M. Stephan, "Light cured networks containing metal organic frameworks as efficient and durable polymer electrolytes for dye-sensitized solar cells,”J. Mater. Chem. A., vol. 21, (2013), pp.9033-9036. 